\title{
Stress fracture of the first rib
}

\author{
M RADEMAKER, AD REDMOND, PV BARBER \\ From Wythenshawe Hospital, Manchester, and Stockport Infirmary, Stockport
}

Among the many patients who present to chest clinics and casualty departments with thoracic pain of obscure origin there are a few in whom the cause is immediately apparent from the initial chest radiograph. We present two examples in young women, who were found to have sustained a stress fracture of the first rib during the course of fairly mild but unaccustomed exertion.

\section{Case reports}

\section{Case 1}

A 21-year-old woman presented to an accident and emergency department complaining that she had had an ache in her left shoulder and above the left clavicle for the previous two days. The pain was continuous but worse on inspiration. Clinical examination showed nothing abnormal and she was not dyspnoeic or cyanosed, nor did she have tachycardia or evidence of deep vein thrombosis. An electrocardiogram was normal. A chest radiograph showed a transverse fracture of the distal end of the first rib (fig 1a). Direct questioning revealed that although she had not sustained direct trauma to her shoulder she had been lifting her 2-year-old daughter using the left arm immediately before the onset of the pain, whereas she would normally have used the right arm for this purpose. It was concluded that a stress fracture of the first rib was the cause of her pain. The left arm was immobilised in a broad sling for one week and she made a rapid and uneventful recovery. A later radiograph showed callus formation at the site of the original fracture (fig 1b), confirming the initial diagnosis.

Address for reprint requests: Dr PV Barber, Wythenshawe Hospital, Manchester M23 9LT.

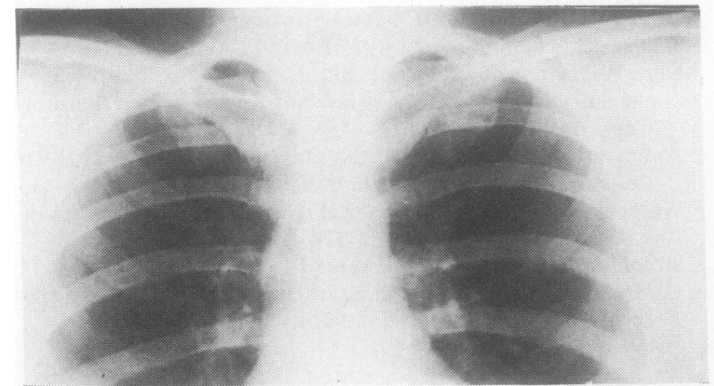

Fig 1 Case 1: (a) transverse fracture of the left furst rib; (b)
Case 2

A 14-year-old girl was referred to the chest clinic by herw general practitioner with a three-week history of pain in the region of the left shoulder and scapula. The pain was worse on deep inspiration. Clinical examination showedw nothing abnormal but the chest radiograph showed a frac- $\infty$ ture of the first rib on the left side (fig 2). Inquiry revealed that she had been working on her father's allotment $\vec{N}$ immediately before the onset of the pain. The work waso strenuous and unaccustomed and had consisted mainly in an attempt to rake some hard, compacted soil. Investigations $\overrightarrow{-}$ (including serum calcium, phosphate, and alkaline phos-0 phatase estimations; liver function tests; full blood count; and ESR) all gave results within normal limits. She was $\overrightarrow{0}$ treated with analgesics. Subsequently she failed to attende for review but was reported to be well and pain free.

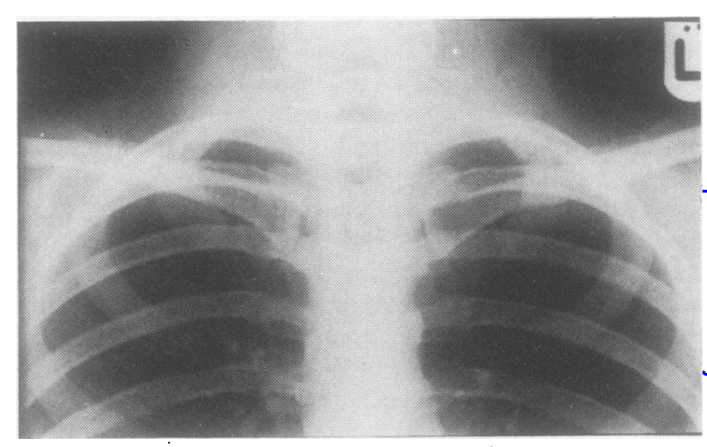

Fig 2 Case 2: fracture of the left first rib.

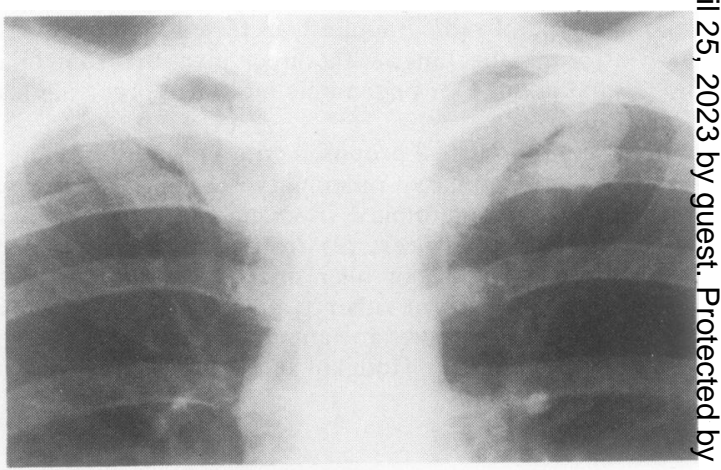




\section{Discussion}

Fracture of the first rib may, of course, follow severe trauma. ${ }^{1}$ Stress fracture of this bone is also well recognised, although for a long time apparent fractures of the first rib were thought to be due to congenital abnormalities of the bone and not acquired. Alderson in a series of 75000 consecutive radiographs of servicemen discovered 73 cases of fracture of the first rib. ${ }^{2} \mathrm{He}$ was able to trace previously normal radiographs in several patients, as well as demonstrating the formation of callus at the site of the original fracture, on subsequent radiographs. In another review Jenkins showed that stress fracture of the first rib occurred predominantly in fit young men, after mild-tomoderate trauma. ${ }^{3}$ Curran and Kelly found that the fracture usually occurs at the weakest point of the rib and that is at the subclavian artery groove immediately posterior to the scalene tubercle. ${ }^{4}$ It has been suggested that it results from a strong contraction of the scalenus anterior muscle combined with traction of the arm. ${ }^{5}$
We report these two cases to illustrate the apparently trivial nature of the exertion leading to the fracture as well as to point out that, although predominantly a male injury, stress fracture of the first rib does occur in healthy young women and must be considered in the differential diagnosis of patients presenting with upper chest and shoulder pain.

\section{References}

' Fordham SD. The significance of first rib fractures. Medical Times 1977; 105:118-9.

${ }^{2}$ Alderson BR. Further observations on fracture of the first rib. $\mathrm{Br}$ J Radiol 1947;20:237,345-59.

${ }^{3}$ Jenkins SA. Spontaneous fractures of both first ribs. J Bone Joint Surg 1942;34:9-14.

${ }^{4}$ Curran JP, Kelly DA. Stress fracture of the first rib. American Journal of Orthopedics 1966;8:16-8.

${ }^{5}$ Aitken AP, Lincoln RE. Fracture of the first rib due to muscle pull. $N$ Engl J Med 1939;220:1063-4. 